\title{
THE ELASTIC-PLASTIC DELAMINATION ANALYSIS OF LAYERED BEAM CONFIGURATIONS
}

\author{
Victor Rizov ${ }^{1^{*}}$ \\ ${ }^{1}$ Department of Technical Mechanics, Faculty of Hydro-technique, University of Architecture, Civil Engineering and \\ Geodesy, 1046 - Sofia, Bulgaria
}

\begin{tabular}{l} 
ARTICLE INFO \\
\hline Article history: \\
Received: 24.2 .2017$. \\
Received in revised form: 29.8 .2017$. \\
Accepted:13.10.2017. \\
\hline Keywords: \\
Fracture analysis \\
Layered beam \\
Elastic-plastic behavior \\
Delamination \\
Analytical solution \\
\hline DOI: http://doi.org/10.30765/er.39.2.6
\end{tabular}

\section{Introduction}

Recently, layered materials have been increasingly applied in various branches of mechanical engineering. This is mainly due to the fact that layered materials are characterized by many useful properties such as high stiffness-to-weight ratio and good process-ability, which makes them a promising alternative to the conventional structural materials.

\begin{abstract}
:
The elastic-plastic delamination fracture in layered beams was studied theoretically. Two Four Point Bend (FPB) beam configurations (the Double Leg Four Point Bend (DLFPB) and the Single Leg Four Point Bend (SLFPB)) were analyzed. An elasticplastic constitutive model with power law hardening was used in the analysis. Fracture behavior was studied by applying the J-integral approach. The analytical solutions of the J-integral were obtained at characteristic levels of the external load. The solutions obtained were verified by analyzing the strain energy release rate with taking into account the material non-linearity. The variation of J-integral value in a function of crack location along the beam dept was investigated. The effect of material non-linearity on the fracture was evaluated. The analysis revealed that the J-integral value decreased with increasing the lower crack arm thickness. It was also found that the material non-linearity has to be taken into account in fracture mechanics based safety design of structural members and components made of layered materials. The analytical solutions obtained are very useful for non-linear investigations, since the simple formulae derived capture the essentials of non-linear fracture in the layered beams under consideration.
\end{abstract}

However, integrity and load bearing capacity of structural members and components composed by the layered materials strongly depend upon their delamination fracture behavior. Delamination, i.e. separation of layers, sharply decreases the stiffness and compressive strength of layered materials. Besides, the post-buckling behavior of layered structures containing delamination cracks under compressive loads can be very complicated.

\footnotetext{
${ }^{*}$ Corresponding author. Tel.: + (359-2) 9635245 / 664; fax: + (359-2) 8656863

E-mail address: v_rizov_fhe@uacg.bg
} 
Obviously, for an efficient and safe design of layered structures, it is necessary to know their delamination behavior. Therefore, the delamination fracture in layered materials continues to attract the interest of researchers [1-8]. Usually, fracture behavior of different layered systems has been investigated by using various beam type configurations, including the FPB beams.

The present paper describes a theoretical study of elastic-plastic delamination fracture in two FPB beam configurations by applying the $J$-integral approach. The analytical solutions of the $J$ integral were obtained at characteristic levels of the external load. The mechanical behavior of the FPB beams under consideration was described by an elastic-plastic constitutive model with power law hardening. It should be noted that the same constitutive model has been applied by the author in non-linear delamination fracture analyses of the Cantilever Beam Opened Notch and the Double Cantilever Beam configurations [9-10]. In the present paper, the $J$-integral solution was derived by using an integration contour that coincides with the beam cross-sections ahead and behind the crack tip. This is in contrast to the analyses developed in [9-10] where the $J$-integral has been solved along an integration contour which coincides with the beam contour. The approach developed in the present paper has some advantages over the analyses presented in [9-10]. For instance, the components of the $J$-integral can be determined in a relatively simple way. Besides, in the present paper, the $J$-integral solution obtained by using the elastic-plastic constitutive model with power law hardening was compared with the strain energy release rate for verification. It should also be mentioned that the present fracture analysis was based on the small strains assumption. The solutions derived were applied to elucidate the effects of crack location along the beam depth and material non-linearity on the delamination fracture behavior.

\section{The elastic-plastic analysis of delamination fracture}

First, the DLFPB beam configuration was analyzed (Fig. 1). There is a delamination crack of length, $2 a$, located symmetrically with respect to the beam mid-span. A vertical notch of dept, $h_{2}$, is cut in the beam mid-span in order to generate conditions for delamination fracture. The DLFPB is loaded symmetrically by two transverse forces, $F$, applied at the beam ends. The beam has a rectangular crosssection of width, $b$, and dept, $h$. The thickness of lower crack arm is $h_{1}$. The upper crack arm is free of stresses.

The fracture was analyzed by applying the $J$-integral written as [11]

$$
J=\int_{\Gamma}\left[U_{0} \cos \alpha-\left(p_{\mathrm{x}} \frac{d u}{d x}+p_{\mathrm{y}} \frac{d v}{d x}\right)\right] d s
$$

where $\Gamma$ is a contour of integration going around the crack tip from one crack face to the other in the counter clockwise direction, $U_{0}$ is the strain energy density, $\alpha$ is the angle between the outwards normal vector to the contour of integration and the crack direction, $p_{x}$ and $p_{y}$ are the components of the stress vector, $u$ and $v$ are the components of the displacement vector with respect to the crack tip coordinate system $x y$, and $d s$ is a differential element along the contour $\Gamma$.

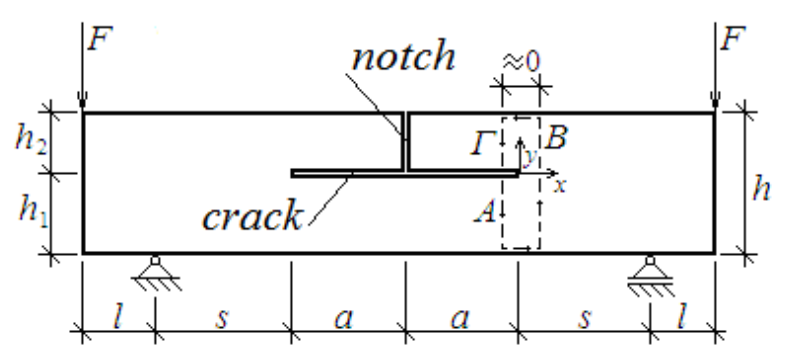

Figure 1. Geometry and loading of the DLFPB beam.

The $J$-integral was solved by using an integration contour that consists of two segments, $A$ and $B$. The segment $A$ coincides with the lower crack arm crosssection behind the crack tip, the segment $B$ coincides with the beam cross-section ahead of the crack tip (Fig. 1).

At low magnitudes of the external load, the beam deforms in linear-elastic stage, i.e. the Hooke's law is valid. The stresses are distributed linearly along the beam depth. Thus, the components of $J$-integral in the lower crack arm cross-section $A$ were written as

$$
p_{\mathrm{x}}=\frac{M}{I_{1}} y_{1}, p_{y}=0
$$


where $I_{1}=\frac{b h_{1}^{3}}{12}$ is the principal moment of inertia of lower crack arm cross-section (here $b$ is the width of beam cross-section). The coordinate, $y_{1}$, varies in the interval $\left[-\frac{h_{1}}{2} ;+\frac{h_{1}}{2}\right], \quad d s=-d y_{1} \quad$ and $\cos \alpha=-1$. The bending moment, $M$, in (2) was obtained as (refer to Fig. 1):

$$
M=F l
$$

The derivative, $\frac{\partial u}{\partial x}$, was written as:

$$
\frac{\partial u}{\partial x}=-\varepsilon_{\mathrm{x}}=-\frac{p_{x}}{E}=-\frac{M}{E I_{1}} y_{1}
$$

where $E$ is the modulus of elasticity. The following formula for the strain energy density was used:

$$
U_{0}=\frac{p_{\mathrm{x}}^{2}}{2 E}=\frac{1}{2 E}\left[\frac{M}{I_{1}} y_{1}\right]^{2}=\frac{M^{2}}{2 E I_{1}^{2}} y_{1}^{2}
$$

By substitution of (2), (4) and (5) in (1), we obtained the following solution of $J$-integral in the cross-section of lower crack arm

$$
\begin{gathered}
J_{A}=\int_{\frac{h_{1}}{2}}^{\frac{h_{1}}{2}}\left\{\left[\frac{M^{2}}{2 E I_{1}^{2}} y_{1}^{2} \cdot(-1)-\frac{M}{I_{1}} y_{1}\left(-\frac{M}{E I_{1}} y_{1}\right)\right]\right\}\left(-d y_{1}\right) \\
=\frac{6 M^{2}}{E b^{2} h_{1}^{3}}
\end{gathered}
$$

The $J$-integral solution in the cross-section $B$ ahead of the crack tip (in the beam un-cracked portion) was obtained by substitution of $h_{1}=h$ in (6). Besides, the sign of (6) was changed to 'minus', since the contour of integration is directed upwards

$$
J_{\mathrm{B}}=-\frac{6 M^{2}}{E b^{2} h^{3}}
$$

The final solution of the $J$-integral was found by summation of (6) and (7) and by doubling the expression obtained in view of the fact that there are two cracks located symmetrically with respect to the beam mid-span (Fig. 1).

$$
J=2\left(J_{\mathrm{A}}+J_{\mathrm{B}}\right)=\frac{12 M^{2}}{E b^{2}}\left(\frac{1}{h_{1}^{3}}-\frac{1}{h^{3}}\right)
$$

It should be mentioned that at $h_{1}=\frac{h}{2}$ the $J$-integral solution (8) coincides with the formula for strain energy release rate obtained when the crack is located in the beam mid-plane [12].

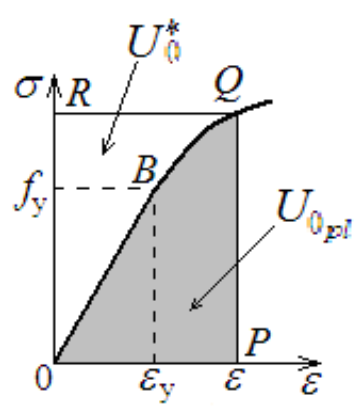

Figure 2. Stress-strain curve of the elastic-plastic model with power law hardening $\left(U_{0_{\mathrm{pl}}}\right.$ and $U_{0}^{*}$ are the strain energy density and complimentary strain energy density, respectively).

Solution (8) is valid at linear-elastic behavior of the beam. However, if the fracture toughness is high, plastic deformation of the beam will begin before the onset of delamination growth from the initial crack tip position. Therefore, the material nonlinearity has to be taken into account in the delamination fracture analysis.

The non-linear fracture in the DLFPB beam configuration was analyzed by using a constitutive model with power law hardening (the corresponding stress-strain diagram is shown schematically in Fig. 2). The linear section, $O B$, of the stress-strain diagram is followed by the non-linear section, $B Q$, with power law hardening in which the stress-strain relation is written as [13]

$$
p_{\mathrm{x}}=\sigma=f_{\mathrm{y}}\left(\frac{\varepsilon}{\varepsilon_{\mathrm{y}}}\right)^{m}
$$

where $f_{\mathrm{y}}$ is the yield stress limit of material, $\varepsilon_{\mathrm{y}}$ is the strain that corresponds to $f_{\mathrm{y}}$, and $m$ is the exponent of power-law. 


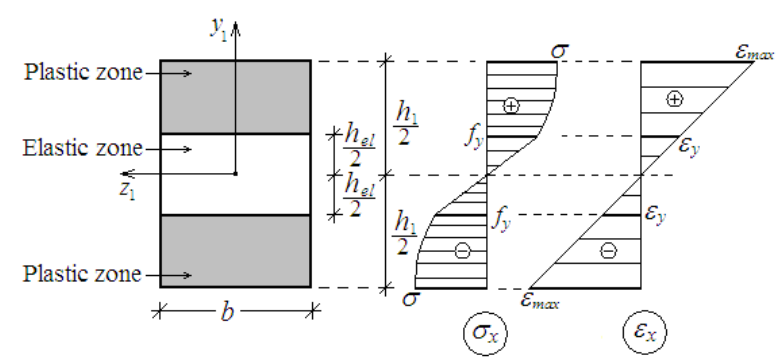

Figure 3. Distribution of stresses and strains in the lower crack arm according to the elastic-plastic model with power law hardening.

At low magnitudes of the external load the beam deforms in the linear-elastic stage and the $J$-integral solution is found by formula (8).

When the external load magnitude increases, the yield stress limit, $f_{\mathrm{y}}$, will be attained first in the remotest edges of lower crack arm with coordinates $y=0,-a \leq x \leq 0$ and $y=-h_{1}, \quad-a \leq x \leq 0$ (Fig. 1). In this case the $J$-integral solution can be obtained by substitution of

$$
M=M_{\mathrm{PL}}=\frac{f_{\mathrm{y}} b h_{1}^{2}}{6}
$$

in (8). The result is

$$
J=\frac{f_{\mathrm{y}}^{2} h_{1}^{4}}{3 E}\left(\frac{1}{h_{1}^{3}}-\frac{1}{h^{3}}\right)
$$

It should be specified that (10) is obtained from formula $f_{\mathrm{y}}=\frac{M_{\mathrm{PL}}}{W_{1}}$, where $W_{1}=\frac{b h_{1}^{2}}{6}$ is the section modulus of lower crack arm cross-section.

Further increase of the external load will lead to appearance of two symmetric plastic zones in the lower crack arm in the portion $-a \leq x \leq 0$ (Fig. 1), while the un-cracked beam portion $(x>0)$ will continue to deform in the linear-elastic stage. The stress distribution in the lower crack arm crosssections is reported in Fig. 3.

The $J$-integral solution in the cross-section of lower crack arm was obtained by integration along the elastic and plastic zones. Thus, the $J$-integral was written as

$$
J_{\mathrm{A}}=J_{\mathrm{el}}+J_{\mathrm{pl}}
$$

where the $J$-integral in the elastic zone of crack arm cross-section was written as

$$
J_{\mathrm{el}}=\int_{\frac{h_{\mathrm{el}}}{2}}^{-\frac{h_{\mathrm{el}}}{2}}\left\{U_{0_{\mathrm{el}}} \cos \alpha-\left[p_{\mathrm{x}_{\mathrm{el}}} \frac{\partial u}{\partial x}\right]\right\} d s
$$

In (13), $h_{\mathrm{el}}$ is the height of elastic zone (Fig. 3), $U_{0_{e l}}$ is the strain energy density in the same zone.

The stresses in the elastic zone are distributed linearly

$$
p_{\mathrm{x}_{\mathrm{el}}}=\frac{M_{2}}{I_{1}} y_{1}
$$

where $M_{2}$ is the bending moment in the lower crack arm $\left(M_{2}>M_{\mathrm{PL}}\right)$.

The partial derivative, $\frac{\partial u}{\partial x}$, was written as:

$$
\frac{\partial u}{\partial x}=-\varepsilon_{\mathrm{x}}=-\frac{1}{\rho_{\mathrm{ep}}} y_{1}
$$

where $1 / \rho_{e p}$ is the curvature of lower crack arm. It should be noted that the present elastic-plastic analysis is based on the Bernoulli's hypothesis for flat beam cross-sections since large span beam to height ratio are under consideration. Concerning the distribution of $\varepsilon_{\mathrm{x}}$ along the height of the beam crosssection, it should also be noted that since the middle portion of the beam (in which the delamination crack is located) is loaded in pure bending (Fig. 1), the only nonzero strain is $\varepsilon_{\mathrm{x}}$. Therefore, according to the small strains compatibility equations, $\varepsilon_{\mathrm{x}}$ is distributed linearly along the height of the beam cross-section.

The equation was used here for equilibrium of elementary forces in the cross-section of lower crack arm in order to determine $1 / \rho_{\mathrm{ep}}$. The equation was written as (Fig. 3)

$$
M_{2}=\frac{b h_{1}^{2}}{2 \varepsilon_{\max }^{2}} \int_{0}^{\varepsilon_{\max }} p_{x}(\varepsilon) \varepsilon d \varepsilon
$$




$$
M_{2}=\frac{b h_{1}^{2}}{2 \varepsilon_{\max }^{2}}\left(\int_{0}^{\varepsilon_{\mathrm{y}}} E \varepsilon \varepsilon d \varepsilon+\int_{\varepsilon_{\mathrm{y}}}^{\varepsilon_{\max }} f_{\mathrm{y}} \frac{\varepsilon^{m}}{\varepsilon_{\mathrm{y}}^{m}} \varepsilon d \varepsilon\right)
$$

After transformations and taking into account the fact that

$$
\varepsilon_{\max }=\frac{h_{1}}{2} \frac{1}{\rho_{\text {ep }}}
$$

equation (17) was written as

$$
\kappa^{2+m}+c \kappa^{2}+d=0
$$

where

$$
\begin{gathered}
\kappa=1 / \rho_{\mathrm{ep}} \\
c=-\frac{2^{m+1}(m+2) M_{2} f_{\mathrm{y}}^{m-1}}{b h_{1}^{2+m} E^{m}} \\
d=\frac{4(m-1) 2^{m} f_{y}^{2+m}}{3 h_{1}^{2+m} E^{2+m}}
\end{gathered}
$$

The curvature, $1 / \rho_{\mathrm{ep}}$, obtained by solution of equation (19) with the help of MatLab computer program has to be substituted in (15) to calculate the partial derivative, $\frac{\partial u}{\partial x}$.

The strain energy density was written as

$$
U_{0_{\mathrm{el}}}=\frac{1}{2} p_{\mathrm{x}_{\mathrm{el}}} \varepsilon_{\mathrm{x}}=\frac{M_{2}}{2 I_{1}} \frac{1}{\rho_{\mathrm{ep}}} y_{1}^{2}
$$

By substitution of (14), (15), and (23) in (13), we obtained

$$
J_{\mathrm{el}}=\frac{M_{2} h_{\mathrm{el}}^{3}}{24 I_{1}} \frac{1}{\rho_{\mathrm{ep}}}
$$

where the height of elastic zone, $h_{\mathrm{el}}$, is determined by using a formula from Mechanics of materials [13-14]

$$
h_{\mathrm{el}}=\frac{2 f_{\mathrm{y}}}{E \frac{1}{\rho_{\mathrm{ep}}}}
$$

The $J$-integral in the plastic zones of lower crack arm cross-section in the portion $-a \leq x \leq 0$ is written as

$$
J_{\mathrm{pl}}=2 \int_{\frac{h_{\mathrm{el}}}{2}}^{\frac{h_{1}}{2}}\left\{U_{0_{\mathrm{pl}}} \cos \alpha-\left[p_{\mathrm{x}_{\mathrm{pl}}} \frac{\partial u}{\partial x}\right]\right\} d s
$$

where the factor of 2 takes into account the fact that there are two symmetric plastic zones in the lower crack arm cross-section (Fig. 3). In (26), $U_{0_{\mathrm{pl}}}$ and $p_{\mathrm{x}_{\mathrm{pl}}}$ are the strain energy density and the stresses in the plastic zones.

The stresses in the plastic zones were obtained by formula (9). The strain energy density in the plastic zones of lower crack arm cross-section is equal to the area, $O P Q$, enclosed by the stress-strain curve (Fig. 2)

$$
U_{0_{\mathrm{pl}}}=\frac{1}{2} f_{\mathrm{y}} \varepsilon_{\mathrm{y}}+\int_{\varepsilon_{\mathrm{y}}}^{\varepsilon} f_{\mathrm{y}}\left(\frac{\varepsilon}{\varepsilon_{\mathrm{y}}}\right)^{m} d \varepsilon
$$

By substitution of $\varepsilon=\frac{1}{\rho_{\mathrm{ep}}} y_{1}$ and $\varepsilon_{\mathrm{y}}=\frac{f_{\mathrm{y}}}{E}$ in (27), we obtained

$$
U_{0_{\mathrm{pl}}}=\frac{f_{\mathrm{y}}^{2}}{2 E}+\frac{E^{m}}{m+1} f_{\mathrm{y}}^{1-m}\left(\frac{1}{\rho_{\mathrm{ep}}}\right)^{m+1} y_{1}^{m+1}-\frac{f_{\mathrm{y}}^{2}}{E(m+1)}
$$

We substituted (9), (15) and (28) into (26). The solution of (26) was written as

$$
\begin{aligned}
& J_{\mathrm{pl}}=\frac{f_{E}^{2}}{E} \frac{1-m}{m+1}\left(\frac{h_{1}}{2}-\frac{h_{\mathrm{el}}}{2}\right)+ \\
& +\frac{m}{(m+1)(m+2) 2^{m+1}} E^{m} f_{\mathrm{y}}^{1-m}\left(\frac{1}{\rho_{\mathrm{ep}}}\right)^{m+1} \times \\
& \times\left(h_{1}^{m+2}-h_{\mathrm{el}}^{m+2}\right)
\end{aligned}
$$


where $h_{\mathrm{el}}$ is obtained by (25).

By substitution of (24) and (29) into (11), we obtained the $J$-integral solution in the lower crack arm

$$
\begin{aligned}
J_{\mathrm{A}}= & \frac{M_{2} h_{\mathrm{el}}^{3}}{24 I_{1}} \frac{1}{\rho_{E}}+\frac{f_{\mathrm{y}}^{2}}{E} \frac{1-m}{m+1}\left(\frac{h_{1}}{2}-\frac{h_{\mathrm{el}}}{2}\right)+ \\
& +\frac{m}{(m+1)(m+2) 2^{m+1}} \times \\
\times & E^{m} f_{\mathrm{y}}^{1-m}\left(\frac{1}{\rho_{\mathrm{ep}}}\right)^{m+1}\left(h_{1}^{m+2}-h_{\mathrm{el}}^{m+2}\right)
\end{aligned}
$$

The $J$-integral solution in the cross-section ahead of the crack tip, $x=+0$, (Fig. 1) was obtained by substitution of $M=M_{2}$ in formula (7). The final solution was found by doubling the sum of (7) and (30) in view of the symmetry (Fig. 1):

$$
\begin{gathered}
J=\frac{M_{2} h_{\mathrm{el}}^{3}}{12 I_{1}} \frac{1}{\rho_{\mathrm{ep}}}++\frac{f_{\mathrm{y}}^{2}}{E} \frac{1-m}{m+1}\left(h_{1}-h_{\mathrm{el}}\right)+ \\
\frac{m}{(m+1)(m+2) 2^{m}} \times E^{m} f_{\mathrm{y}}^{1-m}\left(\frac{1}{\rho_{e p}}\right)^{m+1} \times \\
\quad \times\left(h_{1}^{m+2}-h_{\mathrm{el}}^{m+2}\right)-\frac{12 M_{2}^{2}}{E b^{2} h^{3}}
\end{gathered}
$$

The $J$-integral solution (31) is valid when the bending moment is in the interval

$$
f_{\mathrm{y}} \frac{b h_{1}^{2}}{6}<M_{2}<M_{\mathrm{PLU}}
$$

where the elastic limit load for the un-cracked beam portion is

$$
M_{\mathrm{PLU}}=f_{\mathrm{y}} \frac{b h^{2}}{6}
$$

By introducing $M_{2} / M_{\text {PLU }}$ ratio, formula (32) was re-written in non-dimensional form

$$
\frac{h_{1}^{2}}{h^{2}}<\frac{M_{2}}{M_{\mathrm{PLU}}}<1
$$

When the bending moment reaches the elastic limit load for the un-cracked beam portion, the $J$-integral solution can be found by substituting $M_{2}=M_{\mathrm{PLU}}$ in (31).

If the bending moment magnitude increases further, two symmetric plastic zones will develop also in the un-cracked beam portion, $0 \leq x \leq s$, (Fig. 1). In this case, the solution of $J$-integral in the crosssection of lower crack arm was obtained by substitution of $M_{2}=M_{3}$ in formula (30), where $M_{3}>M_{\text {PLU }}$.

The $J$-integral solution in the beam cross-section ahead of the crack tip was obtained by substituting of $M_{2}=M_{3}, \quad h_{1}=h, \quad 1 / \rho_{\text {ep }}=1 / \rho_{\text {epU }}$ and $I_{1}=I=\frac{b h^{3}}{12}$ in (30). The curvature of un-cracked beam portion, $1 / \rho_{e p U}$, that participates in (30) was obtained from equation (19). For this purpose, $M_{2}=M_{3}$ and $h_{1}=h$ were substituted in (19). Also, $1 / \rho_{\text {ep }}=1 / \rho_{\text {epU }}$ was substituted in (25) when calculating the height of the elastic zone in the beam cross-section ahead of the crack tip, $h_{\mathrm{elU}}$, which was then used in (30). The final solution was found by doubling the sum of the solutions in the crosssections behind and ahead of the crack tip in view of the symmetry conditions (Fig. 1)

$$
\begin{aligned}
& J=\frac{M_{3} h_{\mathrm{el}}^{3}}{12 I_{1}} \frac{1}{\rho_{\mathrm{ep}}}+\frac{f_{\mathrm{y}}^{2}}{E} \frac{1-m}{m+1}\left(h_{1}-h_{\mathrm{el}}\right)+ \\
& +\frac{m}{(m+1)(m+2) 2^{m}} \times E^{m} f_{y}^{1-m}\left(\frac{1}{\rho_{\mathrm{ep}}}\right)^{m+1} \times \\
& \times\left(h_{1}^{m+2}-h_{\mathrm{el}}^{m+2}\right)-\frac{M_{3} h_{\mathrm{elU}}^{3}}{12 I} \frac{1}{\rho_{\mathrm{epU}}}-\frac{f_{\mathrm{y}}^{2}}{E} \times \\
& \times \frac{1-m}{m+1} \times\left(h-h_{\mathrm{elU}}\right)-\frac{m}{(m+1)(m+2) 2^{m}} \times \\
& \times E^{m} f_{\mathrm{y}}^{1-m} \times\left(\frac{1}{\rho_{\mathrm{epU}}}\right)^{m+1}\left(h^{m+2}-h_{\mathrm{elU}}^{m+2}\right)
\end{aligned}
$$


Solution (35) is valid when both the lower crack arm and the un-cracked beam portion deform in elastic-plastic stage, i.e. when the bending moment is higher than the elastic limit load for the uncracked beam portion.

The non-linear solution (35) was verified by performing an analysis of the strain energy release rate with considering the material non-linearity. The strain energy release rate, $G$, associated with a small increase, $\Delta A_{a}$, of the crack area (the external load was kept constant) was expressed as

$$
G=\frac{\Delta W_{\mathrm{ext}}-\Delta U}{\Delta A_{\mathrm{a}}}
$$

where

$$
\Delta A_{\mathrm{a}}=b \Delta a
$$

In (37), $\Delta a$ is a small increase of the crack length. In (36), $\Delta W_{\text {ext }}$ and $\Delta U$ are the changes of external work and strain energy, respectively. The change of external work was written as

$$
\Delta W_{\mathrm{ext}}=\Delta U^{*}+\Delta U
$$

where $\Delta U^{*}$ is the change of complimentary strain energy. By combining of (36), (37) and (38), we derived

$$
G=\frac{\Delta U^{*}}{b \Delta a}
$$

The change of complimentary strain energy was obtained as

$$
\Delta U^{*}=U_{\mathrm{a}}^{*}-U_{\mathrm{b}}^{*}
$$

where $U_{a}^{*}$ and $U_{b}^{*}$ are the complimentary strain energies after and before the increase of crack, respectively. The expression found by combining of (39) and (40) was doubled, because of the symmetry (Fig. 1)

$$
G=2 \frac{U_{\mathrm{a}}^{*}-U_{\mathrm{b}}^{*}}{b \Delta a}
$$

The complimentary strain energy was obtained after the increase of crack by integration of the complimentary strain energy density, $U_{0}^{*}$, in the plastic zones of lower crack arm cross-section (Fig. 3). The strain energy density, $U_{0_{e l}}$, determined by (23) was integrated in the elastic zone of lower crack arm cross-section, since in linear-elastic state the complimentary strain energy density is equal to the strain energy density

$$
U_{\mathrm{a}}^{*}=2 \Delta a b \int_{0}^{\frac{h_{e l}}{2}} U_{0_{\mathrm{el}}} d y_{1}+2 \Delta a b \int_{\frac{h_{\mathrm{el}}}{2}}^{\frac{h_{1}}{2}} U_{0}^{*} d y_{1}
$$

where $U_{0}^{*}$ is equal to the area $O Q R$ that supplements the area $O P Q$ to a rectangle (Fig. 2). Therefore, the complimentary strain energy density was calculated as

$$
U_{0}^{*}=\sigma \varepsilon-U_{0 \mathrm{pl}}
$$

where $U_{0_{p l}}$ is determined by (28).

The complimentary strain energy before the increase of crack was obtained by integration in the crosssection of un-cracked beam portion ahead of the crack tip by using formula (42). For this purpose, $h_{1}$ was replaced with $h$. Besides, $M_{2}=M_{3}$ and $I_{1}=I=\frac{b h^{3}}{12}$ were substituted in (23) in order to calculate $U_{0_{\mathrm{el}}}$.

By combining of (41), (42) and (43), we derived formula for the strain energy release rate that is exact match of the $J$-integral solution (35). This fact verifies the delamination fracture analysis developed by using the elastic-plastic model with power law hardening.

\section{The analysis of the SLFP configuration}

The SLFPB beam configuration is illustrated in Fig. 4. The loading consists of two transverse forces, $F$, applied symmetrically with respect to the beam midspan. 


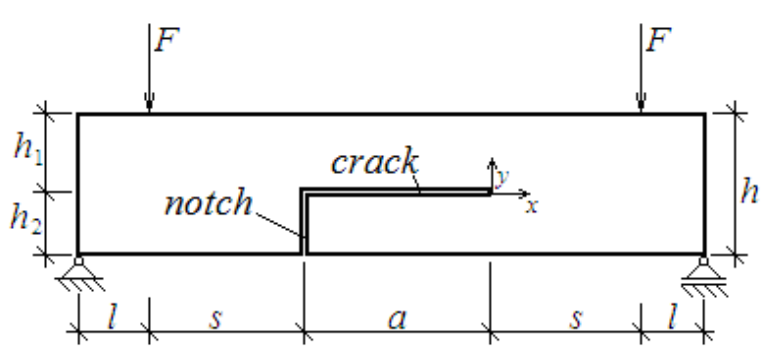

Figure 4. Geometry and loading of the SLFPB beam.

A vertical notch of dept $h_{2}$ is cut in order to induce conditions for delamination cracking. There is one delamination crack of length, $a$. The thickness of the upper crack arm is $h_{1}$. The lower crack arm is free of stress. The beam has a rectangular cross-section of width, $b$, and dept, $h$. It is obvious that all the solutions obtained of the $J$-integral for the DLFPB beam in section 2 of the present paper can be used to analyze linear-elastic, as well as elastic-plastic fracture in the SLFPB configuration. For this purpose, it is necessary only to divide the $J$-integral solutions for the DLFPB by a factor of 2 since in the SLFPB there is only one delamination crack.

\section{Effects of crack location and material non-linearity}

The effect of material non-linearity on delamination fracture behavior of the DLFPB layered beam configuration was evaluated. For this purpose, the $J$ integral non-linear solution (31) was used. Calculations of the $J$-integral value were performed assuming that $f_{y}=1.034 \times 10^{8} \mathrm{~Pa}, b=0.02 \mathrm{~m}$, $h=0.01 \mathrm{~m}$ and $h_{1}=0.0082 \mathrm{~m}$.

The $J$-integral value was presented in a nondimensional form by using the formula $J_{N}=J /(E b)$. The $M_{2} / M_{P L U}$ ratio was varied in the boundaries determined by (34). The $J$-integral value was plotted in the non-dimensional form against $M_{2} / M_{\text {PLU }}$ ratio at $m=0.8$ in Fig. 5. In order to evaluate the influence of elastic-plastic behavior of material on the delamination, the $J$ integral was calculated also by using the linearelastic solution (8) and was plotted in the nondimensional form against $M_{2} / M_{\text {PLU }}$ ratio in Fig. 5 for comparison with the curve generated by elasticplastic solution. The curves in Fig. 5 indicate that

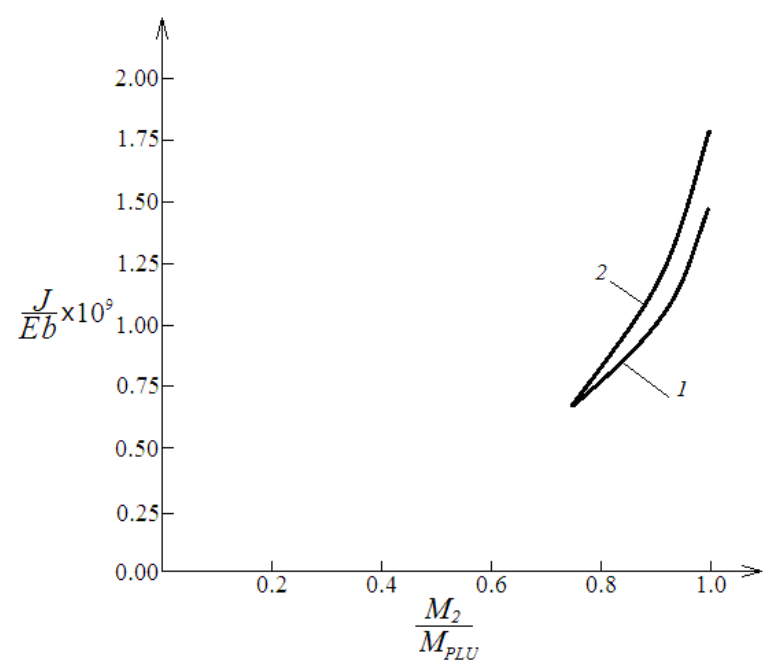

Figure 5. The J-integral value in non-dimensional form plotted against $M_{2} / M_{\mathrm{PLU}}$ ratio (curve 1 linear-elastic behavior of material, curve 2 elastic-plastic behavior of material).

the elastic-plastic behavior of material leads to increase of the $J$-integral value in comparison with the linear-elastic solution. Therefore, the material's elastic-plastic behavior has to be taken into account in fracture mechanics based safety design of structural members composed by layered materials. The influence of a crack location along the beam dept on the elastic-plastic fracture behavior was analyzed too.

For this purpose, the non-linear solution (35) was used. The crack location along the beam dept was characterized by $h_{1} / h$ ratio. The $J$-integral was plotted in non-dimensional form against $h_{1} / h$ ratio at $M_{3}=40 \mathrm{Nm}$ in Fig. 6 . One can observe that the $J$-integral value decreases with increasing of the $h_{1} / h$ ratio. This finding was attributed to the increase of lower crack arm stiffness (the upper crack arm is free of stresses).

\section{Conclusion}

Delamination fracture in the DLFPB and SLFPB layered beam configurations was studied theoretically with taking into account the elasticplastic behavior of material. The fracture analysis was performed by applying the $J$-integral approach. A non-linear constitutive model with power law 


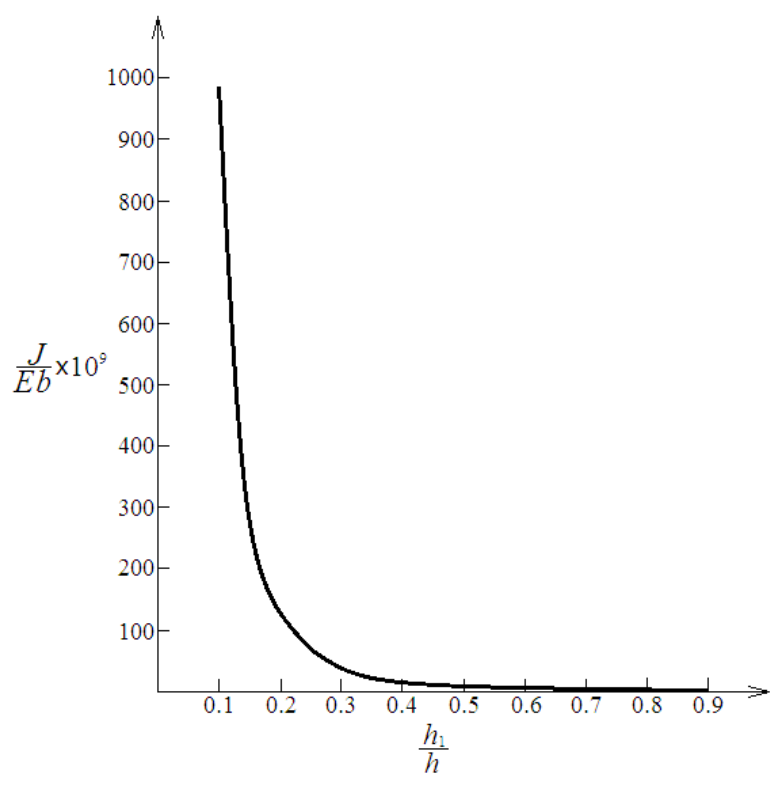

Figure 6. The J-integral value in non-dimensional form plotted against $h_{1} / h$ ratio.

hardening was used to describe the beam mechanical behavior. The analytical solutions of the $J$-integral were derived at characteristic levels of the external load. The solution derived was verified by performing analysis of the strain energy release rate with taking into account the material non-linear behavior. The influence of crack location along the beam dept on the elastic-plastic delamination fracture behavior was analyzed. It was found that the $J$-integral value decreased with increasing $h_{1} / h$ ratio. This finding was attributed to the increase of the lower crack arm stiffness. The effect of material non-linearity on the fracture was investigated too. The analysis revealed that the material non-linearity leads to increase of the $J$-integral value in comparison with the linear-elastic solution. This finding indicates that the material non-linearity has to be taken into account in fracture mechanics based safety design of structural members composed by layered materials. The present paper contributes to the understanding of delamination fracture in layered beams that exhibit elastic-plastic behavior of material.

\section{References}

[1] Jiao, L., Gurumurthy, G.K., Kramer, E.J., Sha, Y., Hui, C.Y., Borgesen, P.: Measurement of interfacial fracture toughness under combined mechanical and thermal stress, J. Electron Packag, 120 (1998), 2, 325-349.

[2] Yeung, D., Lam, D., Yuen, M.: Specimen design for mixed mode interfacial fracture properties measurement in electronic packages, J Electr Packag, 122(2000), 3, 67- 72.

[3] Guadette, F., Giannapoulos, A., Suresh, S.: Interfacial cracks in layered materials subjected to a uniform temperature change, Int J Fracture, 28(2001), 4, 5620-5629.

[4] Tkacheva, L.A.: Unsteady crack propagation in the beam approximation, Applied Mechanics and Technical Physics, 49(2008), 2, 177-189.

[5] Hsueh, C., Tuan, W., Wei, C.: Analyses of steady-state interface fracture of elastic multilayered beams under four-point bending, Scripta Materialia, 60(2009), 1, 721-724.

[6] Her, S.-C., Su, W.-B.: Interfacial fracture toughness of multilayered composite tructures, Strength of Materials, 47(2015), 1, DOI 10.1007/s11223-015-9646-y.

[7] Szekrenyes, A.: Semi-layerwise analysis of laminated plates with nonsingular delamination-The theorem of autocontinuity, Applied mathematical modelling, 40(2016), $1344-1371$.

[8] Szekrenyes, A.: Nonsingular crack modelling in orthotropic plates by four equivalent single layers, European journal of mechanics A/solids, 55(2016), 73-99.

Rizov, V.: Non-linear delamination fracture analysis by using power-law hardening, Multidiscipline Modelling in Materials and Structures, 12(2016), 80 - 92.

[9] Rizov, V.: Non-linear fracture behaviour of double cantilever beam, Engineering Mechanics, 22(2015), 95-102.

[10] Broek, D.: Elementary engineering fracture mechanics. Springer, 1986.

[11] Hutchinson, W., Suo, Z.: Mixed mode cracking in layered materials, Advances in Applied Mechanic, 64(1992), 2, 804-810.

[12] Kishkilov, M.M., Apostolov, R.G.: Introduction in theory of plasticity. UACEG, Sofia, 1984.

[13] Dowling, N.: Mechanical Behavior of Materials. Pearson, 2007.

[14] Zubchaninov, V.: Fundamentals of theory of elasticity and plasticity. Vishaya shkola, M., 1990. 